\title{
IMPLEMENTATION OF CUTTING-EDGE INTERNET TECHNOLOGY TO THE IMPROVEMENT OF REVENUE GENERATION USING CYBER-SECURITY
}

\author{
Oboyi, Joseph ${ }^{1}$, Udeze Chinedu L.*2, Bukie Paul T. ${ }^{3}$, Onoja Emmanuel O. ${ }^{4}$ \\ 1: oboyijoseph@unical.edu.ng, 2: udezechinedu@unical.edu.ng*, 3: \\ bukiepaultawo@unical.edu.ng, 4: onoskiss2006@gmail.com. \\ 1:Department of Mathematics, University of Calabar, Calabar, Cross River State, Nigeria. \\ 2, 3: Department of Computer Science, University of Calabar, Calabar, Cross River State, \\ Nigeria. \\ 4: Department of Cyber Security, Federal University of Technology Minna, Niger State, Nigeria.
}

\begin{abstract}
The need for the most accurate and flexible system of revenue collection from internal sources has become a matter of extreme urgency and importance in e-governance. This need underscores the eagerness on the part of the Government to look for a new principle and policy of revenue collection or to become aggressive and innovative in the mode of collecting revenue from existing sources using the present system. The Boards of some Governments in Africa, even up to the moment are facing a lot of setbacks in performing their tasks due to the manual system of revenue collection from the public. This can be improved through an effective collection of revenue using the most accurate and flexible system. Tax is usually collected in the form of specific sales tax, general sales tax, corporate income tax, individual income tax, property tax and inheritance tax. Problems such as high cost of collection, fraud, underpayment, leakage in revenue, poor access to information, poor tracking of defaulters is at the increase. As a result of this, there is need to computerize the revenue collection system. Computerized systems have proven to introduce massive efficiencies and quick collection of revenue from the public. This research work demonstrates how to design and implement an automated system of revenue collection and how to maintain a secured database for collected tax information. This research delves into the study of how machine learning algorithms and Software-defined Networks improve the security of such automated systems.
\end{abstract}

\section{KEYWORDS:}

Computerized Revenue Collection, machine learning, cyber security, Software defined networks, Object-oriented Programming, Online Database Management.

\subsection{INTRODUCTION}

There stand to be immense benefits in the new computer technologies being implemented today by the developed world. However, there is a great delay in the implementation of such computing technologies in a developing country like Nigeria. In the use of manual systems, records, files, books and related documents are exposed to unauthorized access or damage. It is 
prone to fraud, underpayment or excessive revenue collection. There could also be poor access to information, customers' data and identification of rogue revenue collectors. It could result to high error rate due to bulk volume of work. There are also incidences of fake tax receipts and poor remittance of cash payment. Due to the increase in population, public and private enterprises have increased the rate of tax payment; thereby, posing many problems to the tax collectors who use the door to door and manual system of tax collection.

\subsection{OBJECTIVES AND SIGNIFICANCE OF STUDY}

This research is aimed at the design and implementation of a computerized revenue collection system. Automation of the manual system is geared towards using software systems to keep records of taxable adults and organizations. This will aid in issuing computer-generated receipts. The use of computer databases for management of tax collection information helps in keeping accurate records of transactions and maintenance of customers' data. Computerization will minimize the drawbacks of the error-prone manual system of updating customer status and transactions. The use of computers will increase tax payer compliance and make the process of audit management and verification more error-free. The improved process will improve the computer-literacy of the revenue management staff and personnel. This procedure will enforce equity in collecting the right amount from the customers over the due time frame. This will further reduce the drudgery of labor since a single computer is equipped to replace a number of individuals.

\subsection{LITERATURE REVIEW}

According to [1], effective system of revenue collection and management in the state government constitutes the crucial and central component in the administrative process of the state government. This is largely so because finance determines the services rendered by the state government. [2] proposed the success or failure of any government depends largely on the type of revenue collection system used to generate financial resources through internal revenue available to the individual government and the way those resources are utilized. According to [3], revenue collection system traditionally involves any flexible and accurate process designed to document (keeps record) of customers' transaction, setting prices and controlling capacity to maximize the revenue from a fixed source (internal or external). According to [4], a revenue collection system is a system that is used to bring an effective way of laying charges on users using computer resources, with the aim of recovering the cost of the services that are used, for the purpose of income, maintenance and business continuity.

According to [5], despite the numerous sources of revenue available to all tiers of government in Nigeria apart from petroleum, revenue generation is still at the decline state due to inadequate application of information technology (computers) to the collection processes and management of internally generated revenue. Considering the above, [6] proposed that the collection processes and type of systems used in revenue collection activities depends largely on the technological advancement, population size and the choice of a given government. [47] 
considered revenue collection system as a record which provides details of revenue (income) generated over a given period of time and forecast income analysis.

From the perspective of [7], revenue collection system is any integrated system that can be used to store and retrieve revenue data (information) and at the same time performs cashiering function. [8] described a manual revenue collection system as a system whose process involves the use of filing system to document all revenue activities and transactions either in a cash register or office flat file. Such revenue records are usually manually kept and collection processes and documentation involve mundane tasks. [7] defined a computerized collection system as a system that handles integrated data retrieval system, balance due and non-filer cases requiring telephone communication for resolution. Data stored in the computerized collection system include taxpayers' information and audit details.

[9] the ultimate benefit of computerized revenue collection system is increased revenue. Each resource for the organization is managed to ensure that it generates the maximum possible revenue. Management gains a decision-support resource for marketing and operational functions, having at their disposal a tool that can analyze historical and forecast future revenue management and collection system. According to [10], computerized processing as provided by the electronic system quickens the operations on the input data to produce timely information. He stated further that the degree of accuracy and precision of the information increased by the use of computerized data processing tools, which remove the use of hand-written entries. Computerized or automatic processing is the process of keeping data in a safe place and retrieving them when required via a computer.

According to [11], implementing a computerized revenue collection system posses a lot of challenges on an organization and among these we have: The need for a lot of financial support for the development and maintenance of the system. Political influence in the organization and changes of administration can cause the abandonment of such automation projects. The security of the organization's information becomes prone to breaches and unethical hacking; hence, there could be need for physical and logical cyber-security. The advent of a new software solution can breed the need for more technical expertise in operating and administering the database. It may warrant to the employment of more computer-literate staff and experts. This could also create a need for more physical infrastructure like the construction of server rooms and offices for new technical staff. More so, lack of the knowledge of Information Technology and Software Engineering skills might limit the adoption of such a system.

[12] stated that Information Technology has profoundly changed most organization and their information needs, and revenue accounting must adjust to changes.

According to [11], a computerized revenue collection system provides tax payers the following information: clear, precise and timely information about payment of tax and their records. It ensures adequate response to all tax payers' enquiries. It provides registration forms for all tax payers. It maintains a valid and up-to-date tax payers' record. It alerts taxpayers. According to [7], computerized revenue collection system has been proved to be the most effective and 
efficient method of revenue collection (tax collection) due to its speed, accuracy and lower cost of implementation. Efficient automated systems focus on some key goals which includes security, usability, integrity and sophistication [48].

[14] and [15] proposed some machine learning algorithms to detect DoS, User to Root, Remote to Local and probe attacks. Some researchers have published on the comparison of SDN-based NIDS using several Deep Learning approaches. [16] and [17] used Anomaly Detection while [22] used DDoS Detection System. [19] conducted the comparison using Lightweight DDoS and Flooding Attack while [20] used Intrusion Detection method. [21] performed a research on the implementation of machine learning-based IDS in optical SDN. He categorized attacks into data leakage, data modification, unauthorized, denial of service and security policy misuse. The SDN architecture tries to segregate the network control plane and data plane for easy programmability [23]. [18] explained Internet of Things (IoT) as the network of physical things equipped with the features for computation, communication, storage, software, embedded electronics to enable the objects to gather, process and transmit data. Due to the penetration of IoT into sectors like military, home appliances, automobiles, healthcare and manufacturing, there is need to consider security with enabling technologies like SDN and cloud computing.

There are attackers seeking information from the traffic of internet-based systems such as personal bio-data, business logic details, credentials etc. The open nature of the interface of SDN can make it prone to a host of attacks on applications, IoT devices and infrastructure [24] and [25]. Taxation systems involving Big Data can be hosted on cloud platforms since it handles data processing, resource management and analysis. ML algorithms can be classified into supervised, unsupervised, semi-supervised and reinforcement learning [18]. [26] and [27] have also published some recent works on Deep Reinforcement Learning (DRL). There are varieties of machine learning algorithms used in IoT which includes: NaiveBayes [28], K-Nearest neighbor [29], k-means algorithm [30], Random Forest and Decision Tree [31], Support Vector Machine (SVM) [32], [33], Recurrent Neural Networks (RNN) [34]-[36], Principal Component Analysis [37], Q-learning [30] and Deep Learning [38]. These algorithms can be used in addressing some research problems like authentication, attack detection and mitigation, distributed DoS attack, Anomaly/Intrusion Detection and malware analysis.

Security and privacy can be achieved with some DRL algorithms like prioritized experience replay, asynchronous N-step Q-learning, deep SARSA, deep deterministic policy gradient, asynchronous advantage actor critic, etc [18]. However, ML algorithms in IoT have some limitations such as: limited processing power and energy and poor data management and analytics. Nevertheless, ML security algorithms can be used for authentication and access control [45], especially in IoT applications. These applications can be classified into personal and enterprise. The former includes body area networks, smart office, health-care, sensor networks and smart homes while the latter includes critical infrastructure, smart industries and smart cities [18]. Several researchers have proposed diverse architectures for the security of IoT systems using ML algorithms. Rathore et al. [42] advocated for a semi-supervised learning-based mechanism of attack detection based on Extreme ML. Shi et al. [39] rather advocated for a 
technique of user authentication through WiFi signals based on human physiological activities. Diro et al. [40] by leveraging fog ecosystem advocated for a Deep Learning-based mechanism of attack detection. Abeshu et al. [41] advocated for a distributed DL-based mechanism of detecting attack. ML algorithms are still one of the most reliable means of trapping DDoS attacks in networks.

Li et al. [43] brought up a detection mechanism for DDoS for Cyber-Physical systems while Hodo et al. [45] showed that they could use ANN technique to deal with DDoS attacks. Li et al. [46] equally used an AI-based approach to implement intrusion detection for Software Defined Networks. We try to avert intrusions that can occur through such attacks like masquerading, hijacking control, spoofing etc. Malware are threats that can be executed through a number of attacks which includes virus, spyware, ransom-ware, Trojan, adware etc. Alam et al. [31] was able to detect android-based malware using a random forest classifier with supervised learning method. Azmoodeh et al. [38] worked towards Internet of Battlefield Things (IoBT), a breed of IoT using deep learning to analyze codes from the devices. Karbab et al. [36] devised a tool for Android application framework called MalDozer which is based on deep learning and ANN.

Some of the challenges encountered in using Deep Learning algorithms are: One size does not fit all, neural networks are black boxes, longer time for convergence, butterfly effect of DL and ML, challenges for DL in the edge and vulnerability to security breaches [18]. Even though deep learning is prone to malicious attacks, it can be used for cyber security and in self-driving cars. There are also challenges faced in handling IoT data such as: complicated data collection from diverse domains, proximity effect of mode of data storage, data dependency on learning before problem solving and unavailability of training datasets. Hence, efficient cyber solutions are needed for: complex cyber threats, preservation of privacy in DL- and ML-based solutions, adversarial learning in IoT and ML and lack of awareness and confidence in the security system.

\subsection{RESEARCH METHODOLOGY}

The research methodology adopted in this project work followed the internationally accepted software engineering standard - the Object-Oriented Analysis and Design Methodology (OOADM) with some agile models. Using this method, certain steps were followed for the successful creation of the new system. These steps include: problem identification, feasibility studies, detailed system analysis, system design, program development (coding), implementation and testing and maintenance.

\subsection{Problem Identification and Feasibility Studies}

This is a clear identification and definition of the problems to be studied included scope of the process and overall criteria of effectiveness, efficiency and adaptability. The term "feasibility study" implies a study of the practicality of the proposed project. It involves a preliminary analysis of the total requirements needed for the evaluation of the problems identified. The following types of feasibility studies were carried out by the researcher: 
a. Technical Feasibility study: For the design of this project to be cost effective, the need to consider computerization alternatives cannot be over-emphasized. Possible alternatives include: full computerization, semi-computerization and an improved manual approach. In full computerization, all the systems and sub-systems are computerized with little or no interference. This alternative is very expensive. In semi-computerization, only part of the system is computerized while the other parts are not. In an improved manual approach, no computerization is required; the manual procedures are only improved as a better substitute for the existing system.

b. Economic Feasibility: Carrying out the economic feasibility guarantees that on the completion and implementation of this project, there will be increased effectiveness, usability, efficiency, accuracy, ease of data access and profit maximization of tax collection obtainable in the organization.

\subsection{Detailed System Analysis}

This project was carried out with the aim of contributing to the enhancement and computerization of the revenue collection system. The primary and secondary data sources were combined to form the base of the research methodology used.

\section{Primary Data Source}

Primary data sources are best used to gain direct feedback from individuals and in identifying problems especially in requirement capturing. The primary data source which was used during the course of this research work was face-to-face interviews with members (Staff) of Benue State Board of Internal Revenue Service (BIRS) [13]. During the course of the interviews, the data elements which were needed by the board to carry out their function (collection of revenue) were deduced.

\section{Secondary Data Source}

The secondary data sources were the method most used in collecting information throughout the duration of the project. It was relatively easier to gather reliable information from secondary sources because a lot of background reading was done. The internet also provides valuable sources of articles related to the computerization of revenue collection systems in general.

\subsection{Flow of Data in the Existing System}

Presently, the board uses the Pay Direct Scheme (flat Rate Form) where the taxpayers pay or lodge their assessed tax directly to a collective account after assessment. The taxpayer obtains his/her receipt of tax payment at the collection registry unit after presenting a valid bank teller. The receipt documentation is manually done on a cash register called the office flat file or cash register book. The filing cabinets act as the database. When the need arises to access these records, they are retrieved by means of reaching and collecting the necessary records. The diagram below shows the flow of data in the existing (manual) system. 


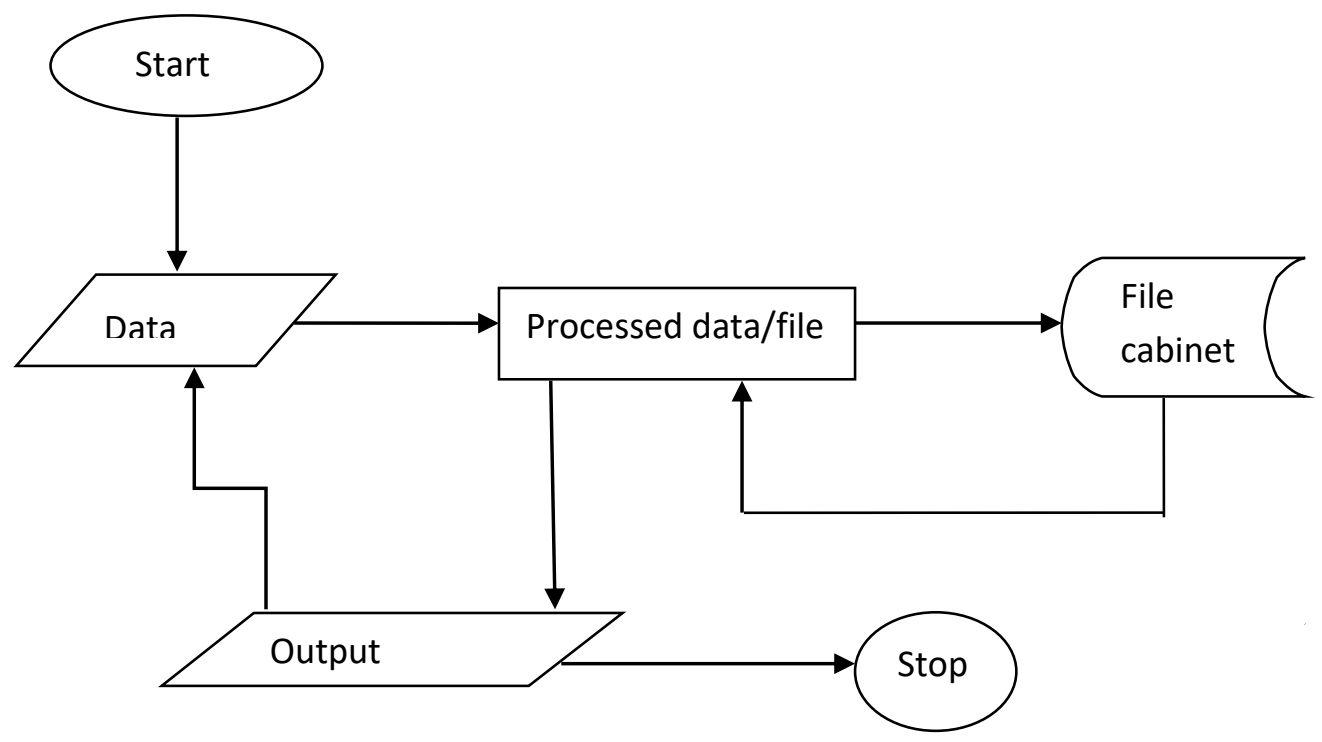

Figure 1 A flowchart depicting the flow of data using the existing system.

\subsection{Functional Requirements}

Functional requirements refer to the functions and features that are going to be implemented in the new system. Some are good features that may be found in the existing system while others will be new features. It is significant to note that these requirements were gathered from the requirement capturing and analysis done earlier. They are invariably the benchmark by which the success of this system development will be measured or assessed. The following are the functional requirements of the new system;

i. The system must provide security measures by usage.

ii. The system must provide a compact system of storage, where all tax files and other revenue transactions are stored on computer hard disk.

iii. The system must generate payment receipts from the database.

iv. A graphical user interface (GUI) might be most appropriate.

\subsubsection{Input Requirements}

The input specification forms the basis of converting the present manual system into a computer based system. The input data for the new system consist of the data consisted in the forms which can be completed in the system. These forms include: tax payment form, tax relief form, tax assessment form, Tax Identification Number (TIN) form and license form.

\subsection{System Design}

The new system will serve as a means of addressing the situation on ground. The system design will be computerized and help in solving the problems associated with the current manual system in use by the board (BIRS). Tasks such as sorting and processing revenue (tax) data at a reduced cost and on increased speed will be performed with relative ease by the new system. The new system component is classified into two (2) major phases which are: The front end and The back end. 


\subsubsection{The Front End}

The front end is made up of;

A. The main screen: The main function of this page is to act as the interface which links the user to the functionalities, operations and pages. The main screen is a stage from which the input and search forms are accessed.

B. System processing: General process of data input into the system.

C. User interface design: The user interface design describes how the software communicates within its reach to a system that interoperates with the software and the humans that use the software. An interface implies a flow of information (e.g. data flow) and specific type of behavior. Therefore, a data flow and flow chart diagram provide much of the information required for the interface design. The user interface consists of log-in page, main page which acts as a link to the other pages and other functionalities of the system.

\subsubsection{The Back End (Database Design)}

Database: this is where all the inputted data (records) are stored for future referencing (or use) and processing. The database consists of different tables that hold all the different categories of data. All information which is collected through the interface is to be stored for future use (or referencing). These pieces of information are stored in the database. The database was designed using the Microsoft Access 2007 which is used to define database and group data attributes and entities into tables. The database is the back end package. The end user can see the database if authorized and can interact with it and perform certain functions permitted via the help of interface. In the design of the database, each form was designated as an entity and therefore had an individual table created under its name, with data input element (field) as its corresponding attributes and given an appropriate data type in the schema design. They include: PayTax table, TaxAssessment table, TaxRelief table, ObtainTaxIdentificationNumber table, TaxPaymentReceipt table, license table and User table. 


\subsection{Flow Chart of the New System}

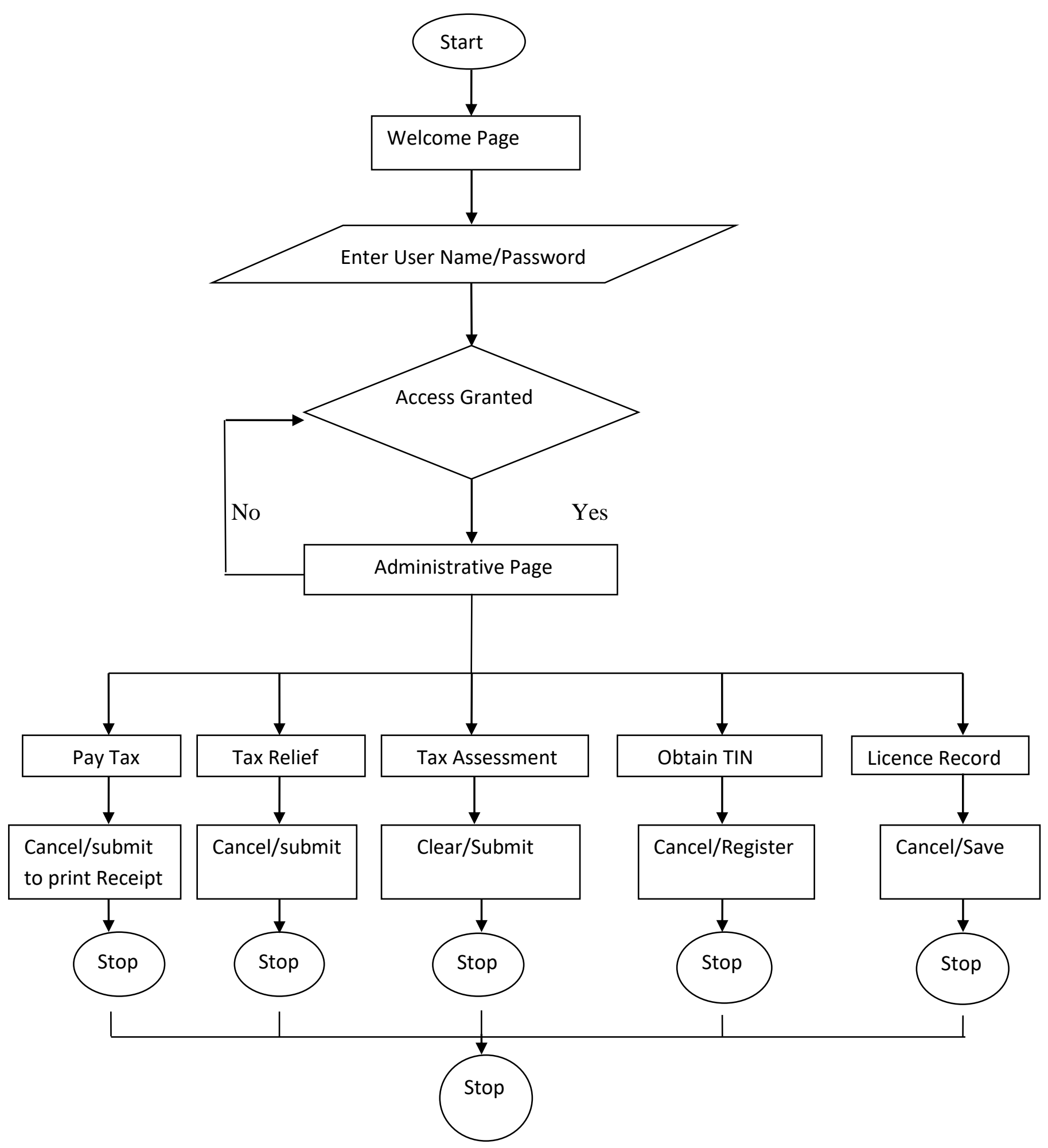

Figure 2: Flow chart diagram of the new system 


\subsection{SYSTEM IMPLEMENTATION AND CODING}

The defined procedures were transformed into control specifications by the help of the programming language. The researcher uses Visual Basic 6.0 for coding the program and Microsoft Access 2007 was used to design the database. Visual Basic 6.0 was used because of its ability to support Component Object Model (COM) and it is the best tool for large storage of data on Personal Computer flat form. Microsoft Access 2007 was used in the design of the database because it allows storage of multiple data and can import or link data directly.

\subsection{Testing}

At this stage, the hardware and software which make up the entire system is tested and thoroughly checked for errors and compatibility. There are three main types of testing carried out on this project. These are:

i. Program Testing: At this stage, the samples of data collected are supplied to the program and output and other reports are generated. Changes are made where necessary and validation and other checking are carried out. The reports and output obtained at the end of this stage are compared with cost implications and other benefits, in other to determine if the new system meets it objectives and hence ensuring its reliability in the operations of the board (BIRS).

ii. System Testing: This stage involves testing the interface between individual parts of the system in overall system test. Here all the physical components that make up the system are tested to ensure that their functionality for the operation for which they were originally intended is achieved.

iii. Acceptance Testing: At this stage, the system is tested by the user department (tax department) that will be working directly with the system. This help to ensure that the new system meets the data processing needs of the organization initially spelt out.

\subsection{Documentation}

This refers to written materials relating to how the system was designed, the way the system works, the way to install the system package and other technicalities to the new system. These written materials could be in form of books, manuals, journals, installation guides and diagram.

\subsection{File Conversion}

This involves converting the previous manual records into a format that is suitable for input/output medium of the new system. It involves the transcription of records into a particular format before they are keyed into the system.

\subsection{Backup Procedure}

The management of BIRS will decide the data backup time; weekly or monthly in order to provide for recovery in case the HARD Disk fails. New backup procedures are available and 
they include; flash drive, CD, Zip drive and others. Regular backup should be provided to ensure that there will be recovery in the event of any disaster.

\subsection{Maintenance}

This involves activities performed to ensure the system is running in good condition. The designer reviews the system from time to time to know the full capabilities of the system and to know its shortcomings based on the latest trends and technologies and if possible to make the required changes.

\subsection{SUMMARY AND CONCLUSION}

We lived in an era of tremendous technological advancement and rapid progress in almost all aspects of living. It is therefore of great importance if the government machinery are computerized to meet the increasing needs and expectations of the public. The designed software is capable of performing the following: capture record of taxpayers through an assessment form, generate Tax Identification Number (TIN), assist in the assessment of taxable income, generate a computerized tax payment receipt and keep license records. It is actually a thing of great importance now that all systems related to office and human procedures are computerized, since it marks for effectiveness and more efficient way of carrying out processes thereby increasing profit at reduced energy cost and provides a better security of data. An organization with computerized systems will in no doubt perform better than those with manual systems; better still, have increased productivity. It is also necessary to adopt novel approaches of machine learning and software defined networks towards the security of such important and financial systems.

\subsection{Recommendations}

The researcher recommends that clients should evaluate this software for the purpose of adding new modules which have been omitted for constraints of finance and time, and go ahead with the implementation of the project. Besides, the board (BIRS) should ensure:

1) Proper training of administrators who shall manage the application especially the area of checking the database.

2) That hardware component is always in proper and good condition. Replacement should be made when there is need.

3) Proper backup of the database be carried out in case of system failure or disaster.

4) As the need of the management increases, the developer should be contacted for software or application upgrade.

\section{CONFLICT OF INTEREST STATEMENT}

On behalf of me and the co-authors, I declare that there is no conflict of interest among the authors. 


\section{SNAPSHOTS FROM THE AUTOMATED SYSTEM}

\section{Figure 3: OBTAIN TAX IDENTIFICATON NUMBER PAGE}

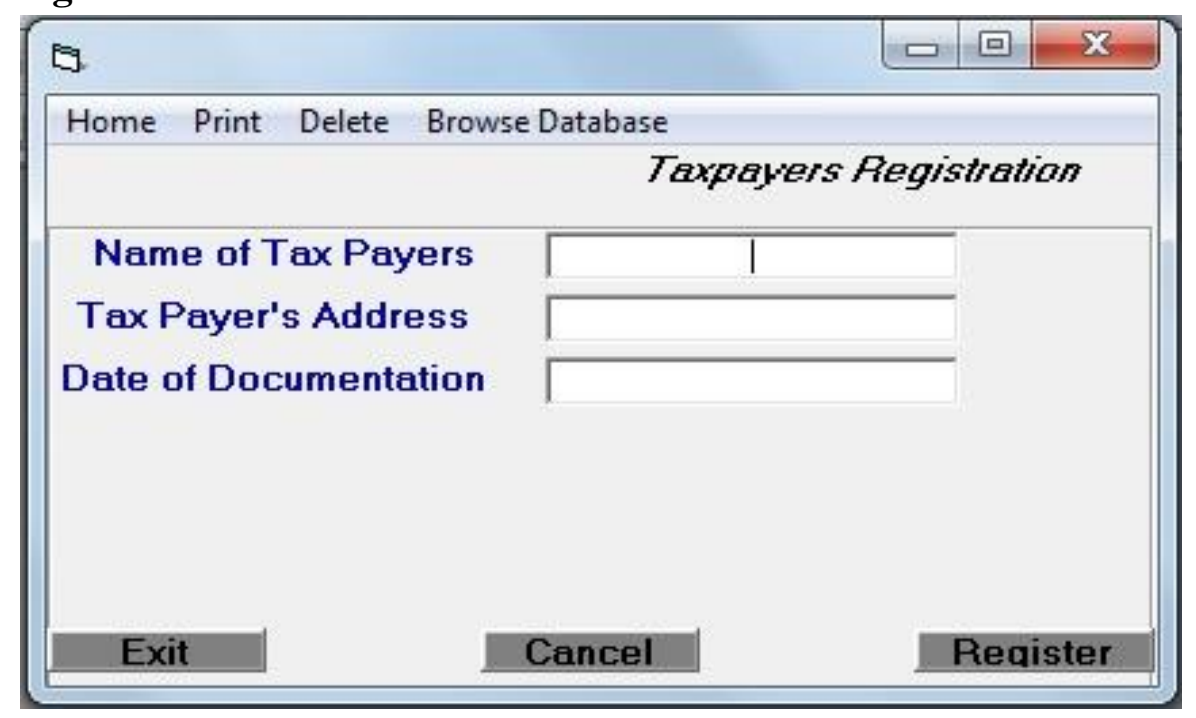

Figure 4: LICENSE PAGE

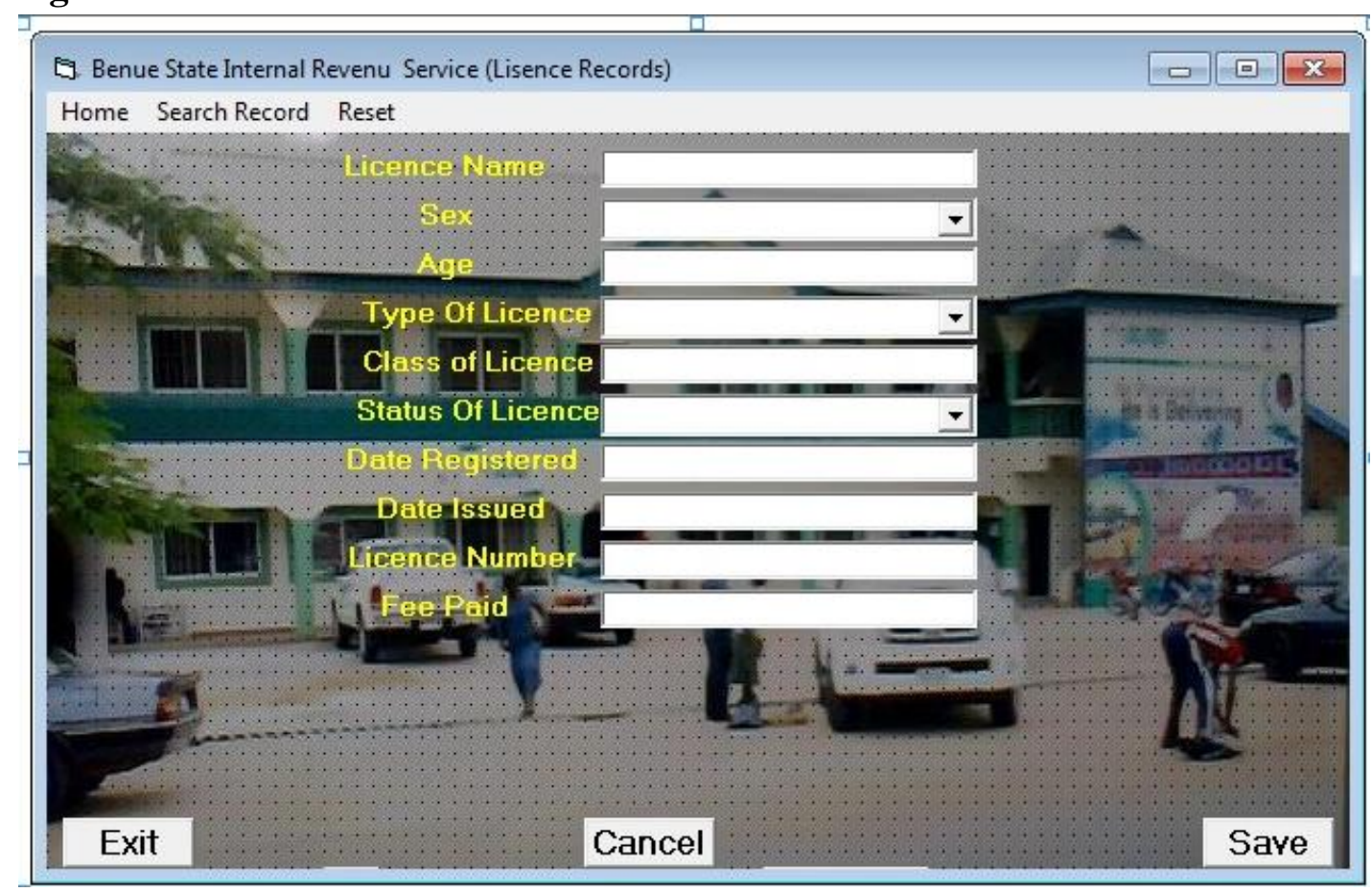

Figure 5: TAX ASSESSEMENT PAGE 


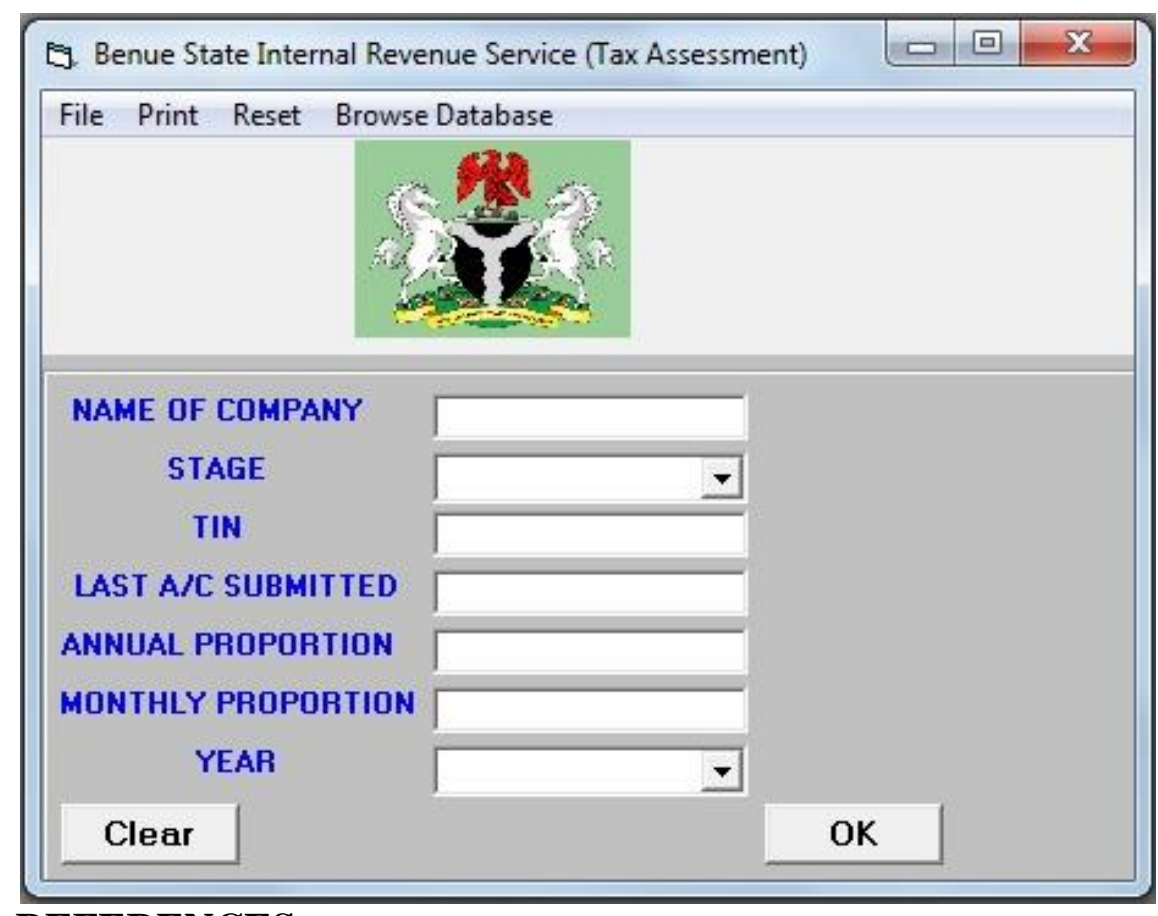

\section{REFERENCES}

1. Ugoo, A. E.: Revenue Collection and State Government Administration in Nigeria, Onitsha, Abbot Book, (2008).

2. Adedeji, A. O.: Public Financial Management. Enugu Malik Enterprises Enugu, Nigeria, (1979).

3. Van, R. G., Gil, J.: Optional Dynamic Actions for Revenue Management. Journal of Economic Literature, 25(2), 699-738 (1987).

4. Bryan, A.: Developing Real Time Application. www.chbmeng.ohio-state.edu/alumni/r (1970).

5. Nwokah, G. N., Kiabel, D. B.: Boosting Revenue Generation in Nigeria: The Tax Consultants Option Revisited. University of Port Harcourt, Nigeria, (2009).

6. Ugunna, E.: Issues in State Government Revenue Collection and Urban Administration in Nigeria. Academic Publishing Company, (1996).

7. Darrin, M.: The Automated Collection System of IRS-Efficient. New York, London, (2006).

8. Guido, S.: Community Revenue Collection System in Malaysia. University of Utara, Malaysia, (2003).

9. RMS - Revenue Management System. http://www.revenuemanagement.com (1997).

10. Saleemi, N. A.: Electronic Data Processing Simplified, $2^{\text {nd }}$ Edition. Saleemi Publishers Nairobi, (1997).

11. Naiwumbwe, B. Z.: An Online Revenue Collection System. A Case Study of Kampala City Council (KCC), (2005).

12. Mohammed, S. A.: Study of the Computerized Revenue Collection System of Local Authority Malaysia. University of Utara, Malaysia, (1998).

13. Benue State Internal Revenue Service: Guidelines on Pay-As-You Earn Scheme as derived from the Personal Income Tax Act 2004 LFN, (2011).

14. Tang, T.A., Mhamdi, L., McLernon, D., Zaidi, S.A.R., Ghogho, M.: Deep learning approach for Network Intrusion Detection in Software Defined Networking. In: 2016 International Conference on Wireless Networks and Mobile Communications (WINCOM), 258-263 (2016). 
IEEE https://doi.org/10.1109/WINCOM.2016.7777224, http://ieeexplore.ieee.org/document/7777224/

15. Latah, M., Toker, L.: Towards an Efficient Anomaly-Based Intrusion Detection for SoftwareDefined Networks, (2018). CoRR http://arxiv.org/abs/1803.06762

16. Mehdi, S.A., Khalid, J., Khaiyam, S.A.: Revisiting Traffic Anomaly Detection using Software Defined Networking. In: Sommer, R., Balzarotti, D., Maier, G(eds) Recent Advances in Intrusion Detection RAID 2011. Lecture Notes in Computer Science vol. 6961. Springer, Berlin, Heidelberg (2011).

17. Tuan, T. A., Mhamdi, L., Mclemon, D., Zaidi, S. A. R., Ghogho, M.: Deep learning approach for network intrusion detection in software defined networking. Int. Conf. Wire1 Netw Mob Commun, (2016). https://doi.org/10.1109/WINCOM.2016.7777224

18. Fatima, H., Rasheed, H., Syed, A. L., Ekram, H.: Machine Learning in IoT Security: Current Solutions and Future Challenges, (2019). ArXiv: 1904.05735v1.

19. Braga, R., Mota, E., Passito, A.: Lightweight DDoS flooding attack detection using NOX/OpenFlow. $35^{\text {th }}$ Annual IEEE Conference on local computer networks, Denver, Corolado, (2010).

20. Jankwoski, D., Ammanowisc, M. On efficiency of selected machine learning algorithms for intrusion detection in software defined networks. Int. J. of Electron Telecommun, 62(3): 247-252 (2016).

21. Nguyen, K. T., Laurent, M., Oualha, N.: Survey on secure communication protocols for the internet of things, Ad Hoc Networks, vol. 32, 17 - 31 (2015).

22. Niyaz, Q., Sun, W., Javaid, A.Y.: A deep learning based DDoS detection system in software defined networking (SDN), (2016). CoRR abs/1611.07400. https://doi.org/10.4108/eai.28-122017.153515

23. Nasrin, S., Naveen, C., Wei, P., Rabei, A.: Survey on SDN based network intrusion detection system using machine learning approaches. Peer-to-Peer Networking and Applications, (2018). https://doi.org/10.1007/s12083-017-0630-0

24. Scott-Hayward, S., O’Callaghan, G., Sezer, S.: Sdn security: A survey. In: 2013 IEEE SDN for Future Networks and Services (SDN4FNS), 1-7 (2013).

25. Dacier, M. C., Konig, H., Cwalinski, R., Kargl, F., Dietrich, S.: Security challenges and opportunities of software-defined networking. IEEE Security and Privacy, vol. 15, pp. 96-100 (2017).

26. Arulkumaran, K., Deisenroth, M. P., Brundage, M., Bharath, A. A.: A Brief Survey of Deep Reinforcement Learning. IEEE Signal Processing Magazine, (2017).

27. Lake, B., Ullman, T., Tenenbaum, J., Gershman, S.: Building Machines That Learn and Think Like People. The Behavioral and Brain Sciences, (2016).

28. Deng, L., Li, D., Yao, X., Cox, D., Wang, H.: Mobile network intrusion detection for iot system based on transfer learning algorithm. Cluster Computing, (2018).

29. Doshi, R., Apthorpe, N., and Feamster, N.: Machine learning ddos detection for consumer internet of things devices. In 2018 IEEE Security and Privacy Workshops (SPW), 29-35 (2018).

30. Hussain, F., Anpalagan, A., Khwaja, A. S., Naeem, M.: Resource Allocation and Congestion Control in Clustered M2M Communication using Q-Learning. Wiley Transactions on Emerging Telecommunications Technologies, (2015).

31. Alam, M. S., Vuong, S. T.: Random forest classification for detecting android malware. In 2013 IEEE International Conference on Green Computing and Communications and IEEE Internet of Things and IEEE Cyber, Physical and Social Computing, 663-669 (2013). 
32. Zhou, W., Yu, B.: A cloud-assisted malware detection and suppression framework for wireless multimedia system in iot based on dynamic differential game. China Communications, vol. 15, 209-223 (2018).

33. Ham, H. S., Kim, H. H., Kim, M. S., Choi, M. J.: Linear svm-based android malware detection for reliable iot services. Journal of Applied Mathematics, vol. 14, p. 10 (2014).

34. Chauhan, J., Seneviratne, S., Hu, Y., Misra, A., Seneviratne, A., Lee, Y. Breathing-based authentication on resource-constrained iot devices using recurrent neural networks. Computer, vol. 51, 60-67 (2018).

35. HaddadPajouh, H., Dehghantanha, A., Khayami, R., Choo, K. K. R.: A deep recurrent neural network based approach for internet of things malware threat hunting. Future Generation Computer Systems, vol. 85, 88 - 96 (2018).

36. Karbab, E. B., Debbabi, M., Derhab, A., Mouheb, D.: Maldozer: Automatic framework for android malware detection using deep learning. Digital Investigation, vol. 24, pp. S48 - S59 (2018).

37. An, N., Duff, A., Naik, G., Faloutsos, M., Weber, S., Mancoridis, S. Behavioral anomaly detection of malware on home routers. In 2017 12th International Conference on Malicious and Unwanted Software (MALWARE), 47-54 (2017).

38. Azmoodeh, A., Dehghantanha, A., Choo K. R.: Robust malware detection for internet of (battlefield) things devices using deep eigenspace learning. IEEE Transactions on Sustainable Computing, 1-11 (2018).

39. Shi, C., Liu, J., Liu, H., Chen, Y.: Smart user authentication through actuation of daily activities leveraging wifi-enabled iot. In Proceedings of the 18th ACM International Symposium on Mobile Ad Hoc Networking and Computing, Mobihoc '17, (New York, NY, USA), ACM, pp. 5:1-5:10 (2017).

40. Diro, A. A., Chilamkurti, N.: Distributed attack detection scheme using deep learning approach for internet of things. Future Generation Computer Systems, vol. 82, 761 - 768 (2018). 41. Abeshu, A., Chilamkurti, N.: Deep learning: The frontier for distributed attack detection in fog-to-things computing. IEEE Communications Magazine, vol. 56, 169-175 (2018).

42. Rathore, S., Park, J. H.: Semi-supervised learning based distributed attack detection framework for iot. Applied Soft Computing, vol. 72, 79 - 89 (2018).

43. Li, Y., Quevedo, D. E., Dey, S., Shi, L.: Sinr-based dos attack on remote state estimation: A game-theoretic approach. IEEE Transactions on Control of Network Systems, 4, 632-642 (2017). 44. Ouaddah, A., Mousannif, H., Elkalam, A. A., Ouahman, A. A.: Access control in the internet of things: Big challenges and new opportunities. Computer Networks, 112, 237 - 262 (2017).

45. Hodo, E., Bellekens, X., Hamilton, A., Dubouilh, P., Iorkyase, E., Tachtatzis, C., Atkinson, R.: Threat analysis of iot networks using artificial neural network intrusion detection system. In 2016 International Symposium on Networks, Computers and Communications (ISNCC), 1-6 (2016).

46. Li, J., Zhao, Z., Li, R., Zhang, H.: Ai-based two-stage intrusion detection for software defined iot networks, CoRR, vol. abs/1806.02566 (2018).

47. Belobaba, P. P.: Direction for Revenue and Management. Journal of Revenue and Pricing Management, 1(1), 87-89 (2002).

48. Udeze, C. L., Umoren, P. U., Oheri, H. E., Attah, H. H.: Automated Students' Results Management Information System (SRMIS). Journal of Multidisciplinary Engineering Science and Technology (JMEST), 4(10), (2017). 